\title{
Efficacy of Corrective Surgery for Gait and Energy Expenditure in Patients with Scoliosis: A Literature Review
}

\author{
Aliyeh Daryabor ${ }^{1,2,3}$, Mokhtar Arazpour $^{1,2}$, Navid Golchin ${ }^{4}$ \\ ${ }^{1}$ Pediatric Neurorehabilitation Research Center, University of Social Welfare and Rehabilitation Sciences, Tehran, Iran \\ ${ }^{2}$ Department of Orthotics and Prosthetics, University of Social Welfare and Rehabilitation Sciences, Tehran, Iran \\ ${ }^{3}$ Student Research Commute, University of Social Welfare and Rehabilitation Sciences, Tehran, Iran \\ ${ }^{4}$ Department of Neurosurgery, Firoozgar Hospital, Iran University of Medical Sciences, Tehran, Iran
}

The aim of this literature review was to evaluate selected original papers that measured gait parameters and energy expenditure in idiopathic scoliosis (IS) treated with surgical intervention. IS is a progressive growth disease that affects spinal anatomy, mobility, and left-right trunk symmetry. Consequently, IS can modify human gait. Spinal fusions remain the primary approach to correcting scoliosis deformities, thereby halting progression. Using the population intervention comparison outcome measure framework and selected keywords, 15 studies that met the inclusion criteria were selected. Alteration of spatial and temporal variables in patients with IS was contradictory among the selected studies. Ankle and foot kinematics did not change after surgery; however, pelvic and hip frontal motions increased and pelvic rotation decreased following surgery. Patients with IS continued to show excessive energy expenditure following surgery in the absence of a physical rehabilitation protocol. Spinal surgery may be considered for gait improvement and IS treatment. There were inadequate data regarding the effect of corrective surgery on the kinetics, energy expenditure, and muscle activity parameters.

Keywords: Scoliosis; Surgery; Fusion; Gait; Walking; Energy expenditure

\section{Introduction}

Idiopathic scoliosis (IS) is termed as such due to the unknown etiology of the deformity. It is a complex deformity in which the trunk deviates from its normal plane of symmetry, inducing geometric changes to the spine in three dimensional space [1]. The prognosis, risk of curve progression, and treatment for IS are based on the remaining extent of spinal growth [2]. Treatment options for the prevention of IS progression include exercises, application of cast or braces, and surgery. The appropriate treatment choice depends on the severity of the deformity and the type of curve [3].

For patients whose scoliosis progresses, spinal surgery (fusion) is the optimal treatment for correcting and stabilizing the deformity, thereby maintaining as many mobile spinal segments as possible and halting progression $[4,5]$. Adolescents with IS are the most common population of patients to receive this type of treatment; however, a considerable population of adults experiences degenerative

Received Nov 4, 2017; Revised Feb 24, 2018; Accepted Apr 17, 2018

Corresponding author: Mokhtar Arazpour

Department of Orthotics and Prosthetics, University of Social Welfare and Rehabilitation Sciences, Kodakyar St., Daneshjo Blvd., Evin, Tehran, 1985713834, Iran

Tel: +98-21-22180010, Fax: +98-21-22180049, E-mail: M.arazpour@yahoo.com 
changes due to IS who are candidates for spinal fusion [6]. This fusion is frequently expanded from the thoracic region into various portions of the lumbar spine and can be administered via the anterior, posterior, or a combination of both sides. The efficacy of surgery on pain, trunk form, and the decompensation phenomenon, regardless of surgical procedures, has been well documented. Following surgery, the spine stiffens, with reductions in the spinal range of motion (ROM) [7].

Walking is an essential activity for human body, and its efficiency depends on muscle activity, joint motion, body coordination, and the ability to adjust the center of gravity $[8,9]$. Given that the trunk assists in the maintenance of balance [10], the presence of a spinal deformity can alter the center of mass (COM) movement during gait, leading to the development of a pathological gait. Walking patterns notably differ between individuals with untreated IS and their healthy peers. These differences include decreased step length [11-13], decreased cadence [14], reduced ROM in the lower extremities [11], and excessive energy expenditure [15].

Several studies have been published explaining the changes in gait parameters for adolescents and adults with IS before and after surgical intervention, but to the best of our knowledge, no comprehensive review in this regard has been published to date. Therefore, the purpose of this review was to evaluate the temporal-spatial, kinetic, kinematic, electromyography (EMG), and energy expenditure parameters in patients with IS before and after spinal surgery.

\section{Methods}

\section{Search strategy}

The search strategy was based on the population intervention comparison outcome method and included all relevant articles published between 1980 and 2017. The following databases were searched: PubMed, Science Direct, Google Scholar, Scopus, and ISI web of knowledge using the words "OR," "AND," and "NOT." Keywords related to the condition included scoliosis, scoliotic, and idiopathic scoliosis; keywords related to the intervention included surgery and fusion; and keywords related to the outcome measures included gait, walking, walking speed, walking velocity, kinetic, kinematic, cadence, step length, stride length, step width, stride width, spatiotemporal, temporas- patial, energy expenditure, energy cost, electromyography, and $E M G$. Ultimately, 15 articles were selected for final evaluation. Two abstracts were included because there was no accompanying full text $[16,17]$. Only English language articles were included. The procedure was followed using the preferred reporting items for systematic reviews and meta-analyses methods (Fig. 1).

\section{Inclusion and exclusion criteria}

This review contains those articles that evaluated the effect of spinal fusion as an intervention method on gait parameters and/or energy expenditure in adult and adolescent patients with IS. Studies that involved the use of other interventions simultaneously (such as spinal braces/

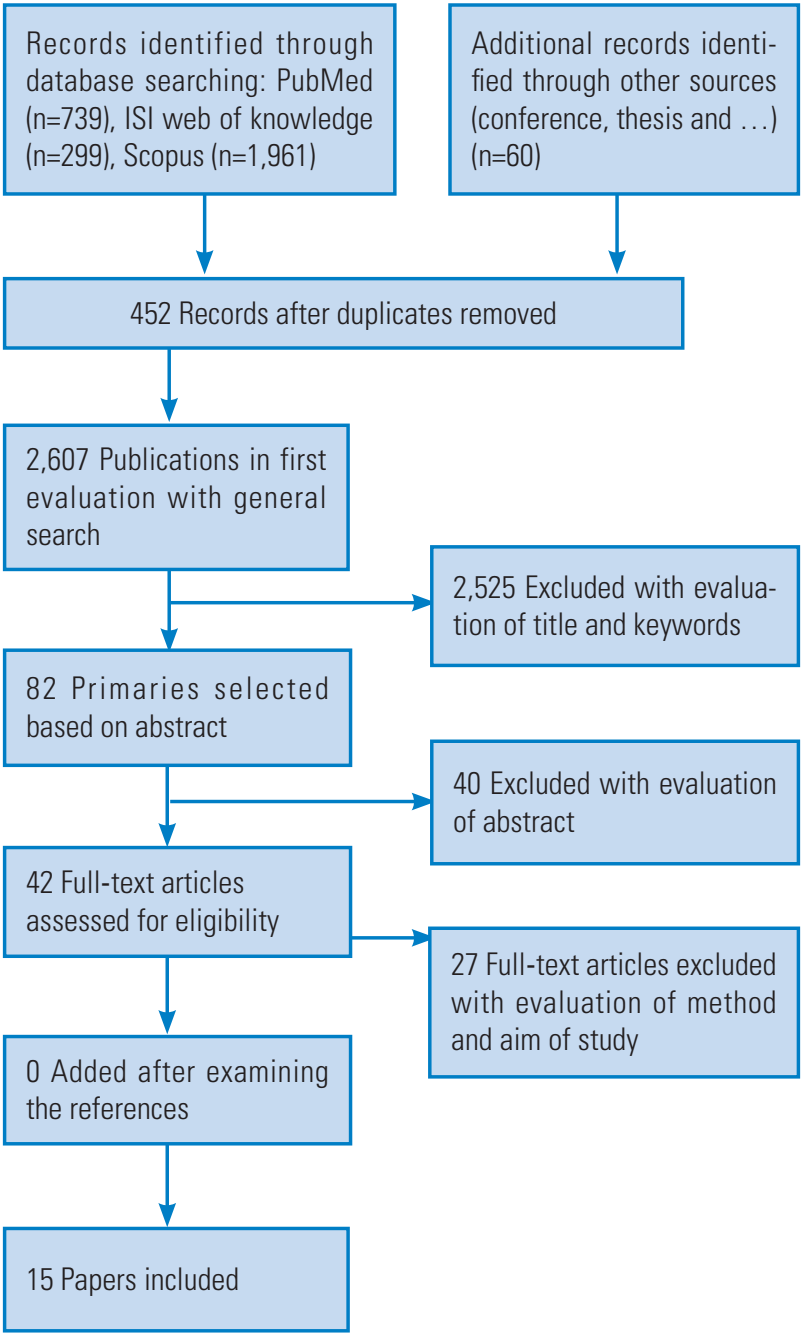

Fig. 1. The procedure was followed using the PRISMA (preferred reporting items for systematic reviews and meta-analyses) method. 
Table 1. Inclusion and exclusion criteria

\begin{tabular}{|c|c|}
\hline Inclusion criteria & Exclusion criteria \\
\hline $\begin{array}{l}\text { Prospective studies including controlled clinical trials (randomized clinical trials), clinical } \\
\text { trials, cross-over, and/or case series }\end{array}$ & $\begin{array}{l}\text { Studies related to the effect of spinal braces on } \\
\text { outcome measure }\end{array}$ \\
\hline $\begin{array}{l}\text { Studies related to the effect of spinal surgery on outcome measures (temporal-spatial, kine- } \\
\text { matic, kinetic, electromyography and energy expenditure parameters) during gait in adult } \\
\text { and adolescent with the idiopathic scoliosis }\end{array}$ & Studies that evaluated postural balance \\
\hline $\begin{array}{l}\text { The study population consisted of adult and adolescent with idiopathic scoliosis who all } \\
\text { underwent spinal fusion surgery }\end{array}$ & Studies that evaluated other types of scoliosis \\
\hline Studies that compared surgery intervention (post-surgery) with pre-surgery condition & Studies where the population had other disabilities \\
\hline Studies that compared surgery intervention (post-surgery) with norm & $\begin{array}{l}\text { Studies which involved use of other intervention } \\
\text { (e.g., orthosis) }\end{array}$ \\
\hline
\end{tabular}

orthoses, functional electrical stimulation, and casts) as well as those studies in which the participants presented with other disabilities (e.g., neurological/musculoskeletal disorders) were excluded. Moreover, studies examining other types of scoliosis, such as congenital, neuropathic, traumatic, and myopathic scoliosis, were excluded. Table 1 provides further details regarding the inclusion and exclusion criteria.

\section{Results}

The following text summarizes the results obtained by studies investigating the effects of the surgical intervention on gait parameters and energy expenditure in patients with scoliosis (Tables 2-5). We reported the effect of scoliosis deformity for each gait variable in patients with scoliosis and in normal controls. The qualitative and quantitative data are shown in Table 2 and Tables 3-5, respectively.

\section{Temporal-spatial parameters}

\section{1) Gait velocity}

Gait velocity parameter was measured in ten articles (Tables 2, 3). Three studies by Engsberg et al. [6,18,19] reported significant reductions in gait velocity between postoperative conditions and the healthy group. In five studies, gait velocity was not significantly altered following surgery [17-21].

In a study by Lenke et al. [7], gait velocity was significantly decreased between the preoperative test $(1.29 \mathrm{~m} /$ $\mathrm{sec}$ ) and the two postoperative tests (1 year, $1.20 \mathrm{~m} / \mathrm{sec} ; 2$ years, $1.19 \mathrm{~m} / \mathrm{sec}$ ). In one study, velocity significantly im- proved in adults with IS after surgery [22]. Engsberg et al. [19] have shown that gait velocity for the primary group improved at 2 years after corrective surgery, such that it was not different from the healthy participants.

\section{2) Cadence}

Patients with IS showed significantly lower preoperative gait cadence compared with the healthy participants $[6,17,22]$. Although some studies demonstrated that preoperative cadence was lower than the able-bodied cadence, there was no significant difference between them $[16,19]$. Moreover, two studies did not find significant differences between controls and IS group when measuring cadence $[16,20]$.

Comparing preoperative and postoperative sessions, four studies reported that cadence was significantly reduced in patients with IS after spinal fusion surgery $[7,17,18,23]$. In one study, cadence was demonstrably modified immediately after surgery $(p<0.05)$, but there was no significant difference between the pre- and postoperative values at 3, 6, and 12 months [16]. Moreover, Yagi et al. [22] and Holewijn et al. [24] have reported that surgery did not have a significant effect on cadence (Tables $2,3)$.

\section{3) Stride/step length}

Compared with the healthy participants, patients with IS showed significantly shorter preoperative walking stride length $[19,22]$. Two studies did not find significant differences between the control and IS group when measuring stride length $[16,20]$. In another study, stride length was significantly shorter in the revision group compared with that in the primary group [6]. 


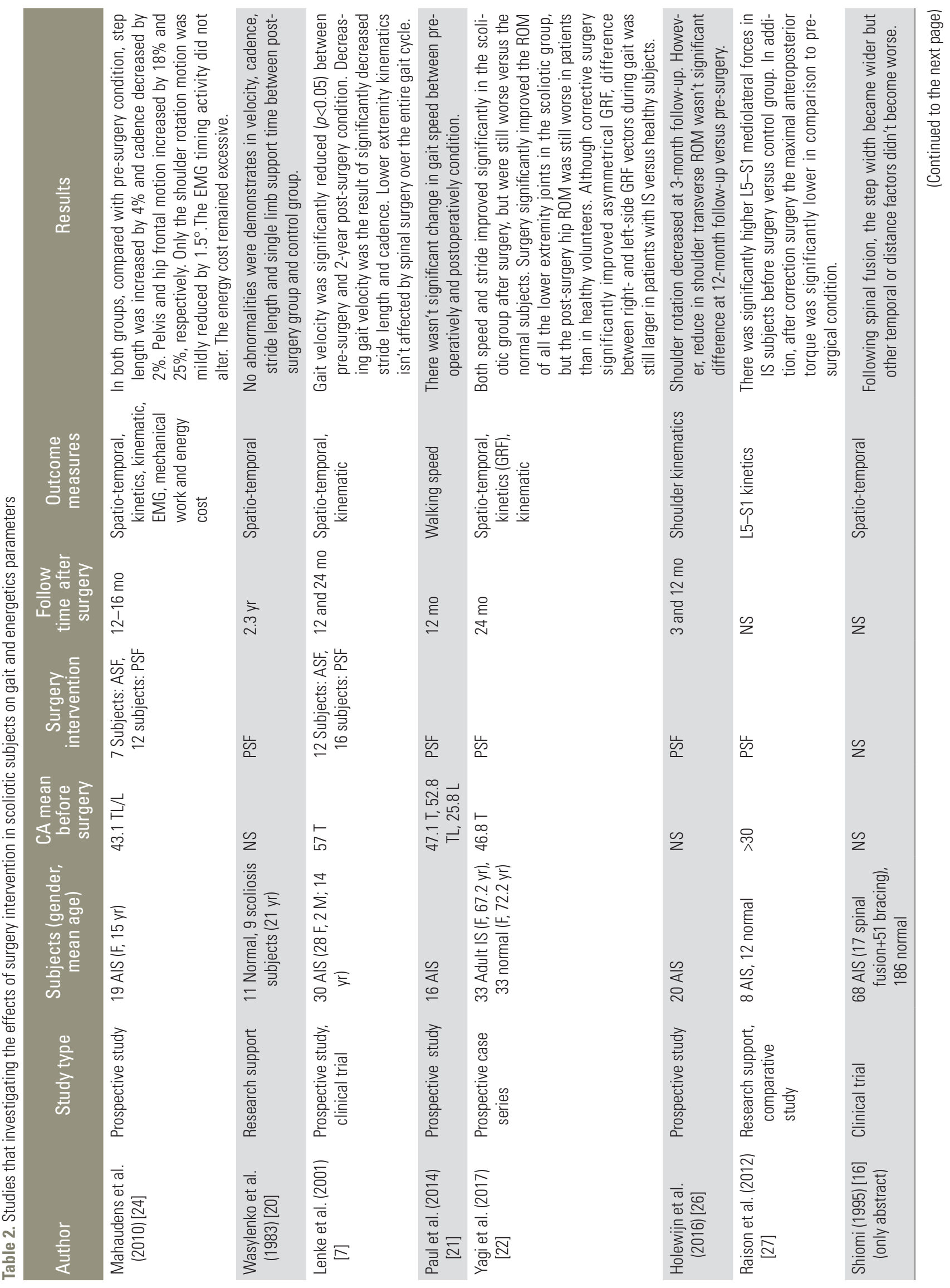




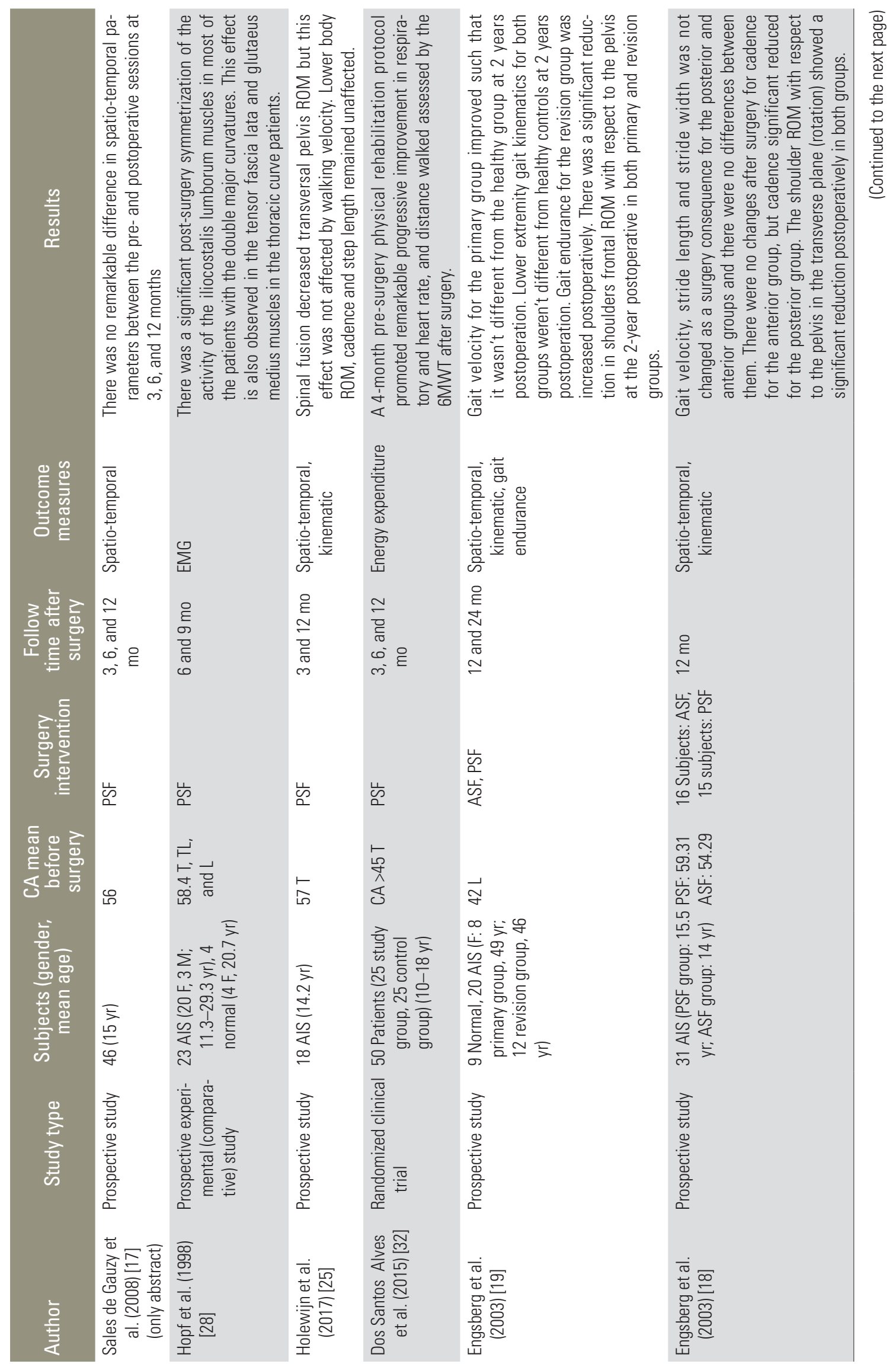


Comparing the preoperative and postoperative conditions, Mahaudens et al. [23] and Yagi et al. [22] have demonstrated that there was a significant increase in stride length (preoperative: 1.32 and $0.97 \mathrm{~m}$ versus postoperative: 1.38 and $1.07 \mathrm{~m}$, respectively). However, Lenke et al. [7] have reported that stride length had significantly decreased at 2 years postoperatively from that preoperatively (preoperative, 1.28; 2 years postoperative, 1.24). In another study, stride length was modified immediately after surgery $(p<0.05)$, but there was no significant difference between the pre- and postoperative values at 3,6 , and 12 months [16]. Holewijn et al. [24] and Yagi et al. [22] have reported that surgery did not have a significant effect on stride length (Tables 2, 3).

\section{4) Stride/step width}

Four studies evaluated the effect of surgical treatment on stride/step width when walking in patients with IS (Tables 2,3 ); however, the results were contradictory. Lenke et al. [7] have demonstrated a significant decrease in stride width preoperatively and at 12 -months postoperatively, but not at 2 years postoperatively. Two studies have shown that there were no changes in stride width postoperatively compared with that preoperatively $[6,18]$. In another study, step width became wider after spinal fusion [16].

\section{Kinematic parameters}

Kinematic parameters were measured in eight articles that focused on motion and ROM of the pelvis and lower extremities (Tables 2, 4).

\section{1) Kinematics of the pelvis and lower limbs}

Comparing the postsurgery and able-bodied groups, the results of two studies have revealed that surgical correction significantly improved the lower extremity gait kinematics in the patients with IS to the point that they were no longer different from the healthy volunteers $[19,22]$.

Comparing preoperative and postoperative sessions, five studies have shown that ankle and foot kinematics did not change after surgery $[7,19,22-24]$. In a study by Mahaudens et al. [23], frontal pelvis and hip motions increased 18\% (preoperative 6.6 versus postoperative $7.8, p=0.04$ ) and $25 \%$ (preoperative 9 versus postoperative 11.3, $p=0.005$ ), respectively. Holewijn et al. [24] have reported the sagittal hip ROM was significantly increased at 3 months after surgery $\left(44.7^{\circ} \pm 3.4^{\circ}\right.$ versus $\left.46.8^{\circ} \pm 2.8^{\circ}\right)$, but this was no longer 

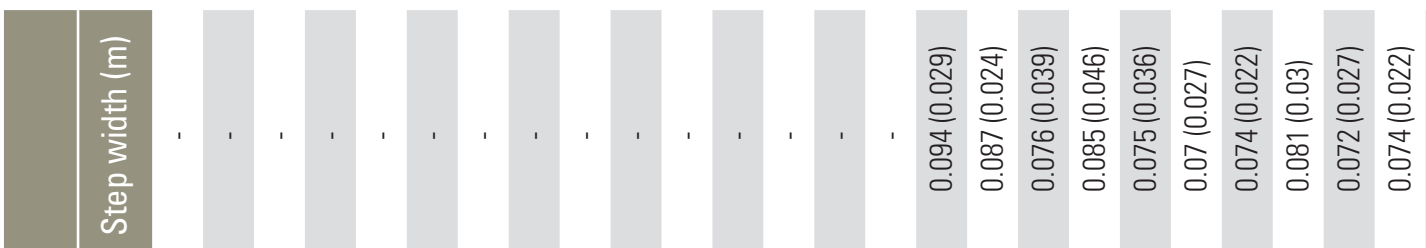

छ

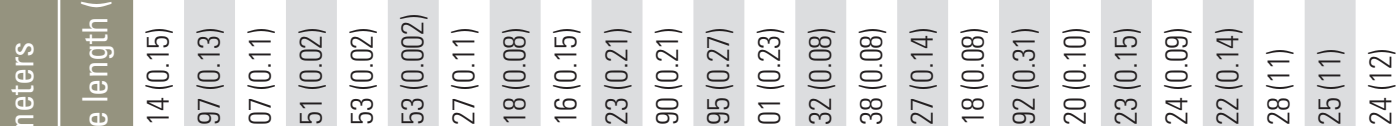

咅

舟

$\frac{\sqrt{\pi}}{\frac{\pi}{5}}$

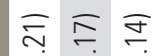

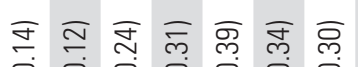

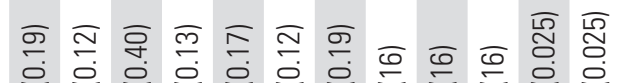

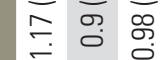

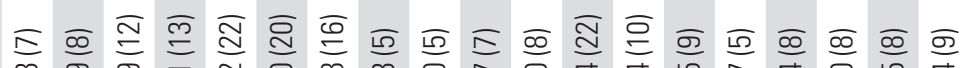

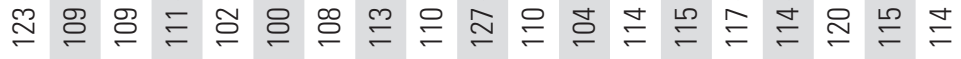

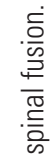

m.

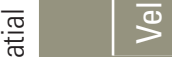

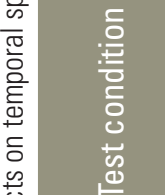

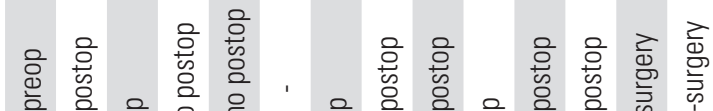

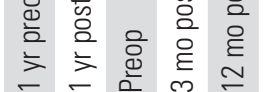

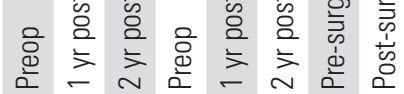

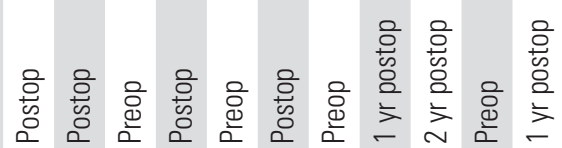

$\frac{\sqrt[4]{2}}{20}$

는

음

든

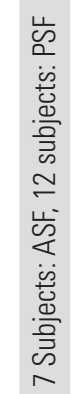

岩

$\subseteq$



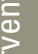

$\sum_{\bar{d}}^{\bar{d}}$

$\stackrel{2}{2}$

के के

똠

菂

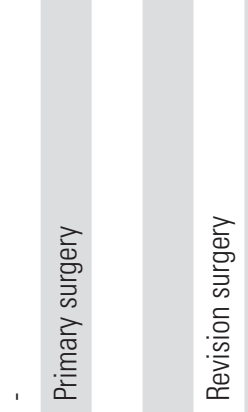

密

m

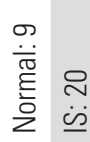

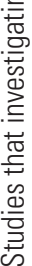

iv

$\stackrel{\infty}{\frac{\infty i j}{i}}$

몬

$\stackrel{\circ}{\frac{\pi}{\pi}} \approx$

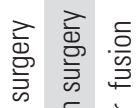

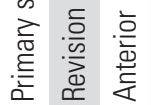

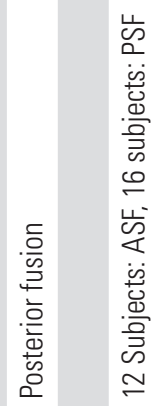

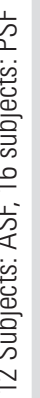

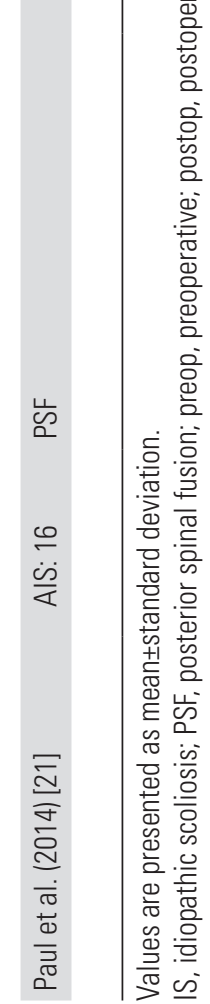




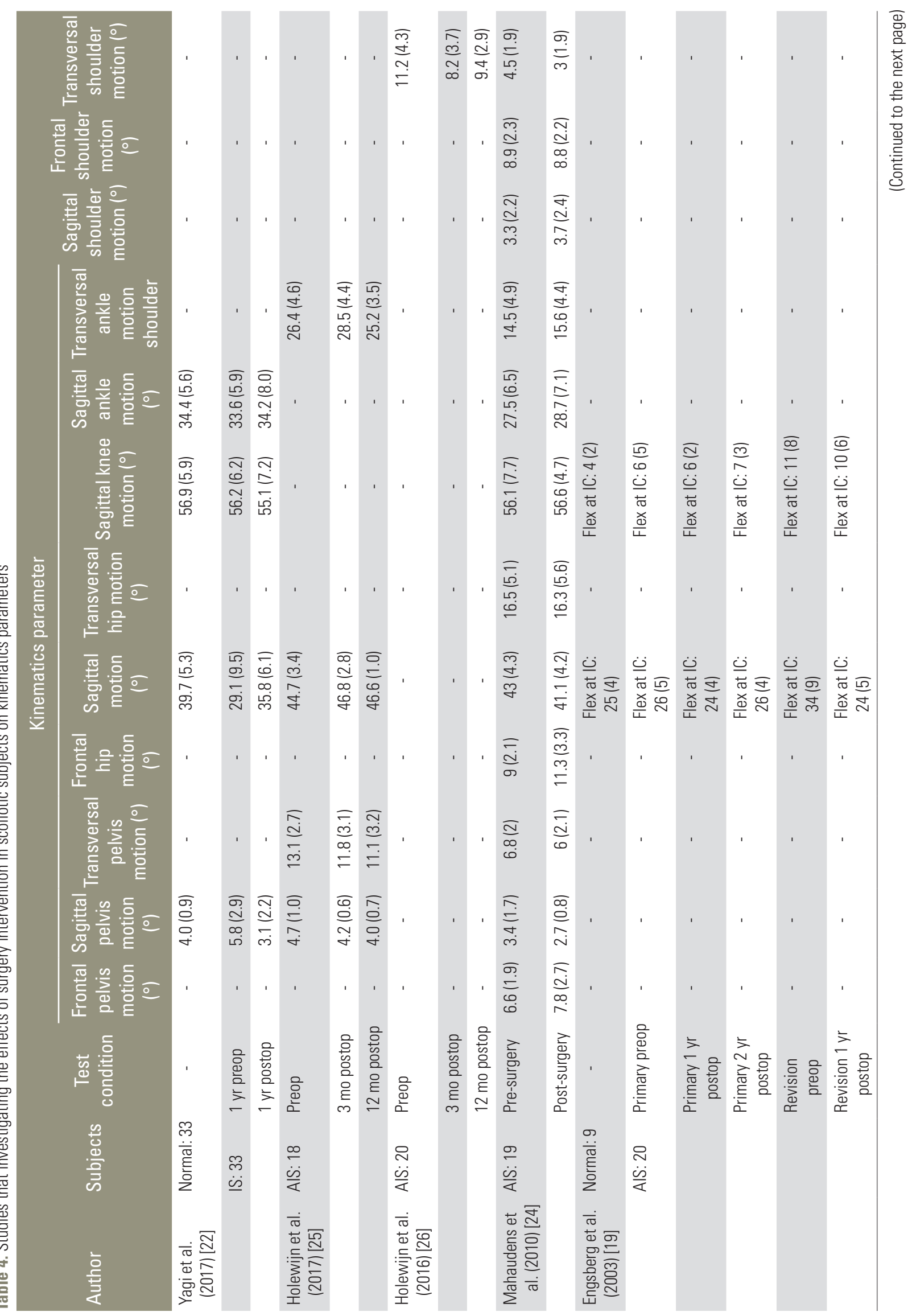




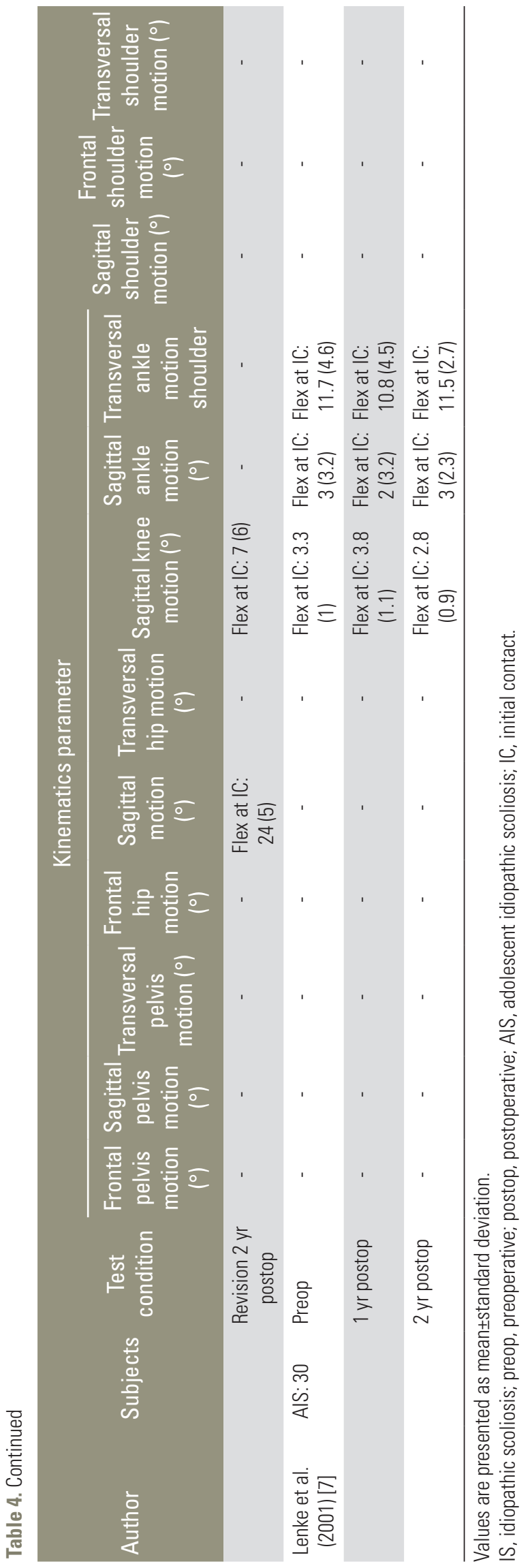

significant at 12 months after surgery $\left(46 \cdot 6^{\circ} \pm 1 \cdot 0^{\circ}\right)$. In addition, spinal fusion decreased transversal and sagittal pelvis ROM at 3 and 12 months postoperatively; however, this effect was not affected by speed [24].

\section{2) Kinematics of the shoulder}

Transverse shoulder motion was reduced by $33 \%$ (preoperative $4.5^{\circ} \pm 1.9^{\circ}$ versus postoperative $3^{\circ} \pm 1.9^{\circ}, p=0.04$ ) in one study [23]. In the same article, shoulder ROM in the transverse plane after surgery decreased by $3^{\circ}$ at 3-month follow-up; however, this decrease was not significantly different at 12-month follow-up compared with the preoperative motion of $9.4^{\circ} \pm 2.9^{\circ}$ [25]. In both articles, shoulder ROM in the sagittal and frontal planes did not significantly change following surgery $[23,25]$.

Shoulders with respect to pelvic kinematics were measured in three articles by Engsberg et al. $[6,18,19]$. The results of three studies generally showed that the shoulders relative to the pelvis had greater flexion in the primary and revision groups compared with that in the ablebodied controls. In addition, variables for the coronal and sagittal planes were lower than those of the healthy volunteers. However, some of these differences were not statistically significant. The shoulder ROM with respect to the pelvis in the transverse plane indicated a significant decrement postoperatively [18]. There was a significant decrease in the shoulder frontal ROM with respect to the pelvis at 2 years postoperatively in both the primary and revision groups [19].

\section{Kinetics parameters}

Only two studies reported the effect of reconstruction surgery on kinetic variables in patients with IS (Table 2). In an article by Yagi et al. [22], patients with IS had asymmetrical ground reaction force (GRF) walking in the vertical and mediolateral direction compared with healthy controls. Although corrective surgery for these patients significantly improved these parameters, differences between right- and left-side GRF vectors during gait were still greater in the patients with IS versus the healthy participants [22]. Raison et al. [26] have reported significantly higher L5-S1 mediolateral force in patients with IS before surgery than in typically developed adolescents. In addition, after corrective surgery, the maximal anteroposterior torque was significantly lower than that before surgery [26]. 

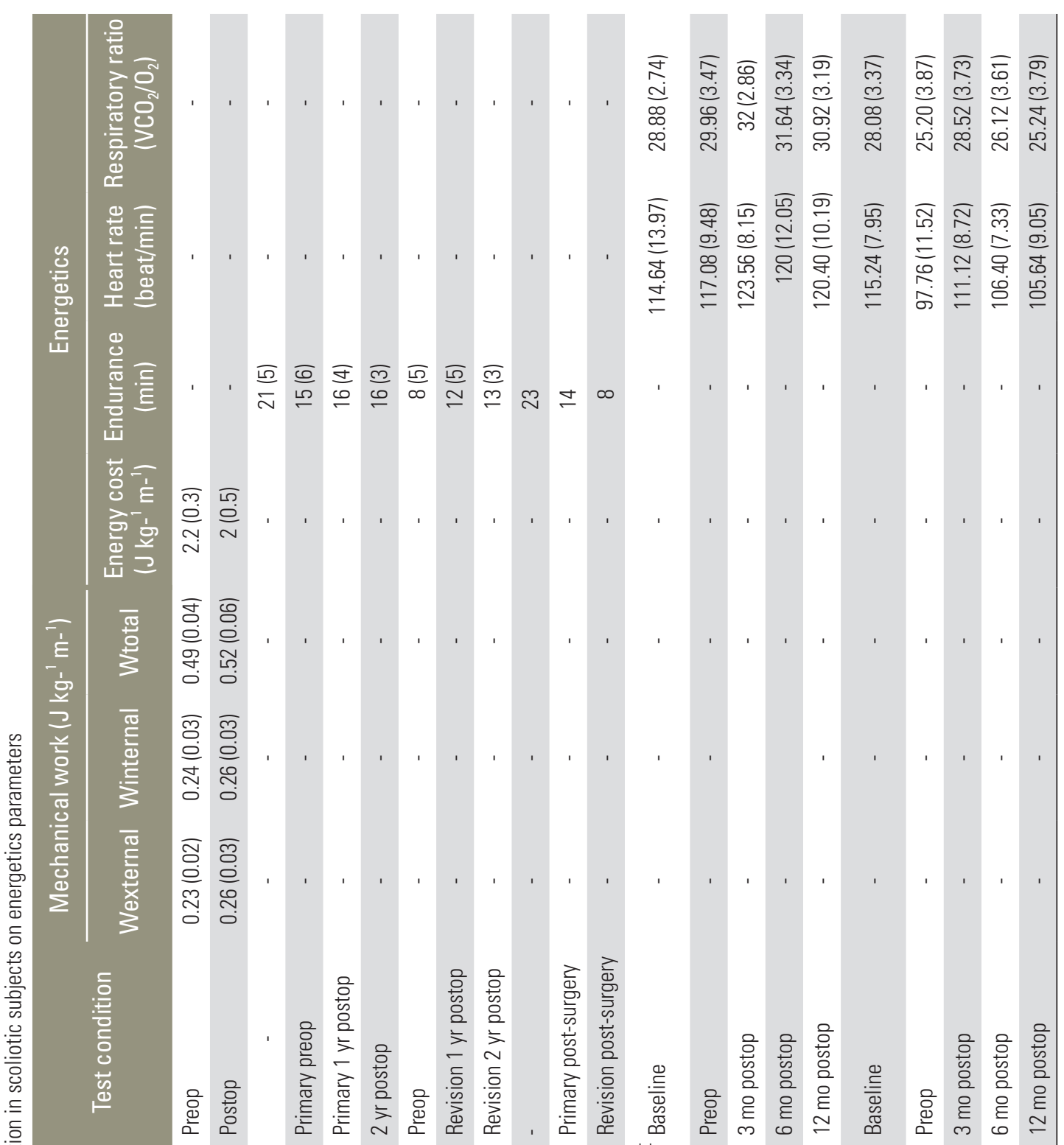

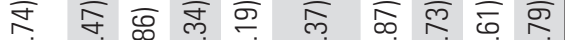

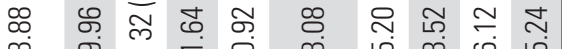

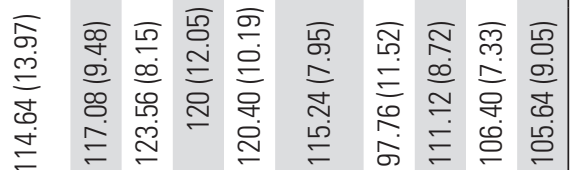

它

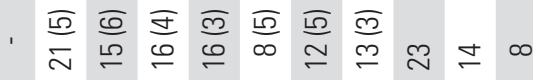




\section{Electromyography}

Only two studies analyzed muscle activity during gait (Table 2). In a study by Mahaudens et al. [23], the EMG duration of the lumbo-pelvic muscles did not change after corrective surgery. Another study by Hopf et al. [27] has found a statistically significant reduction $(p<0.05)$ in the activity of the tensor fascia lata and gluteus medius muscles of the concave side of thoracic scoliosis as well as of the lumbar muscles of the convex side of double major curvatures before and after surgery.

\section{Mechanical work}

Mechanical work was analyzed in one study (Tables 2, $5)$. In this case, $\mathrm{W}_{\text {total }}$ was significantly increased by $6 \%$ $(p=0.02)$ after surgery, mainly due to $\mathrm{W}_{\text {external, }}$ which increased by $13 \%$ (preoperative $0.23 \mathrm{~J} / \mathrm{kg} / \mathrm{m}$ versus postoperative $0.26 \mathrm{~J} \mathrm{~kg} / \mathrm{m}, p<0.001$ ) [23].

\section{Energy expenditure}

Energy expenditure in patients with IS was measured during gait by the evaluation of $\mathrm{O}_{2}$ cost $(\mathrm{mL} / \mathrm{kg} / \mathrm{min}), \mathrm{O}_{2}$ consumption $(\mathrm{mL} / \mathrm{kg} / \mathrm{min})$, the physiological cost index (beat/ $\mathrm{m})$, heart rate (HR, beat $/ \mathrm{min}), \mathrm{O}_{2}$ uptake $(\mathrm{L} / \mathrm{min})$, peripheral oxygen saturation $\left(\mathrm{SpO}_{2}\right)$, and respiratory ratio (RR) during walking $[15,28-30]$. One variable that was used to evaluate the graded exercise endurance test was the duration a person walked on the treadmill until attaining his/ her target endpoint of $70 \%$ to $75 \%$ of predicted maximum HR $[6,19]$.

Four studies have reported the effect of surgery on energy expenditure (Tables 2, 5). Mahaudens et al. [23] have demonstrated that energy expenditure before surgery showed a tendency toward a reduction compared that after surgery; however, no significant differences were noted between the two conditions in patients with IS. The results for the endurance test showed that the revision group demonstrated a significant increase in gait endurance of the revision group at the 2-year postoperative session [19], but their endurance remained less than that of the ablebodied and primary groups $[6,19]$. There was no significant change in endurance for the primary group between the preoperative test session and both the 1- and 2-year postsurgical test sessions. There was a tendency toward a reduction between the primary and able-bodied groups; however, no significant difference was reported $[6,19]$. In a randomized clinical trial (RCT), the effect of a 4-month pre-surgery physical rehabilitation protocol was evaluated for HR, RR, and distance walked by the 6-minute walk test (6MWT) in patients with IS after surgical correction compared with matched controls without physical rehabilitation. The results showed that the physical rehabilitation protocol significantly improved HR, RR, and walking distance after surgery. For $\mathrm{SpO}_{2}$, there was no difference between groups [31].

\section{Discussion}

This literature review was developed to evaluate the impact of spinal surgery on gait parameters and energy expenditure in patients with IS.

\section{Temporal-spatial parameters}

Alterations to walking speed, stride length, and cadence in patients with IS were contradictory among studies. These contradictory results might be due to the difference among the studies in the severity of spinal deformity [32], curve location in the spine [33], and postural stability control of body center in patients with IS [34]. Following spinal fusion, gait results demonstrated no change in gait velocity, stride length, or stride width in several studies [17-21]. In contrast, evidence has shown that reduced gait velocity after surgery relative to that before surgery as well as that of able-bodied individuals is a result of both a reduced cadence and stride length [6,7]. It is unclear whether this is due to restriction from the spinal fusion placed on the trunk and torso or to a possible deconditioning effect in these patients, who are potentially less active after spinal fusion surgery than before [7]. Both the primary and revision groups showed compromised gait velocity and stride length. The revision patients were more compromised than the primary patients [6]. Evidence suggests that primary fusion surgery improved gait velocity such that it was no different from that of able-bodied individuals at 2 years postoperatively. Therefore, any improvement in gait velocity is likely related to the altered mechanics of the patient's spinal alignment and pain improvements that are achieved by surgery [19]. Conversely, one paper has reported an increase in stride length at higher velocities. Moreover, despite reductions in pelvic ROM at higher gait velocities, step length was not decreased and cadence 
was not increased [24]. Another study has noted that gait speed, cadence, and stride length are not directly correlated with walking endurance [22].

\section{Kinematic parameters}

The results for kinematic parameters showed an increase in sagittal hip ROM (3 months postoperatively, but not 12 months postoperatively) [24], an increase in pelvic and hip frontal ROM [23], a decrease in transversal, sagittal pelvis ROM [24], and a decrease in transverse shoulder ROM postoperatively compared with those preoperatively. The increase in pelvic and hip frontal motion following surgery could be explained by the necessity to compensate stiff girdle dissociation with better frontal ROM in the unaffected joints, i.e., the hips and pelvis [23]. Another reason could be that corrective surgery for both the curve deformity and frontal imbalance eliminates the need for excessively careful gait strategy with limited ROM, such as that preoperatively [11]. The hip sagittal ROM improved for at least 2 years after surgery in one study, but it remained impaired compared with that of healthy individuals [22]. Possible reasons found for the limited after surgery ROM of the hip joint were the effect of pelvis and lumbosacral fusion and weak muscle strength in patients with IS [22]. In another study, however, hip flexion at initial contact for both the primary and revision surgery groups improved such that they were not different from that for the able-bodied group 2 years postoperatively [19]. The discrepancy among these studies could be due to differences in the participants' impairments or to the methodologies employed. On the contrary, the pelvic and hip compensations that are necessary for these patients before surgery might not be required after surgery in association with gait training. This possibility should be explored in further studies, including the association between scoliotic patients' training and improvement in these measured parameters. For the ankle and foot kinematics, no changes were reported in these parameters regarding gait after surgery $[7,19,22-24]$.

\section{Kinetic parameters}

Very few studies focus on the impact of spinal surgery on gait kinetic variables. Scoliosis severity can result in abnormal internal joint movements along the spine during walking. Such internal joint movements can cause a sup- plementary asymmetric vertebral growth modulation. In addition, surgical correction reduced maximal anteroposterior torque during gait [26]. The results of the GRF parameter presented an abnormality of vertical GRF vector and asymmetry of the right and left vertical and mediallateral GRFs in patients with IS before surgery. The GRF parameters improved in patients with IS after surgery; however, they remained poorer than those in the healthy controls [22]. Further studies are needed regarding the effect of surgical intervention on exerted movements in the joints of patients with IS during gait.

\section{Electromyography}

Scarce research has analyzed EMG following surgery in patients with IS. Preoperative asymmetry of muscle activation is a result of an asymmetric geometry of the upper body with a consequent asymmetric muscle force pattern. Hopf et al. [27] have noted that restoring the geometric symmetry of the body following surgery creates the corresponding symmetry of the pertinent muscle forces. In another study, the EMG timing activity of the lumbo-pelvic muscles did not change gait after surgery [23] although there was no comparison with normal controls in these two studies. Additional studies are required to analyze EMG gait following surgical correction in patients with IS.

\section{Mechanical work and energy expenditure}

During walking, we not only move our lower legs and pelvis, but also lift our COM down and up at each step. Hip and pelvic frontal motions are essential determinants that minimize the vertical displacement of COM [35]. This vertical displacement of COM plays a vital role in optimizing $\mathrm{W}_{\text {total }}$ (i.e., the work induced by the whole body's muscles to move it through space) [35], and as a result, allow a decrease in metabolic cost [36]. To walk with a muscular mechanical effort that is higher or lower than normal increases the energy expenditure of the gait [37]. IS $\mathrm{W}_{\text {total }}$ has been reported to increase after surgery. The increase in $\mathrm{W}_{\text {total }}$ was primarily due to an increase in external work and could be explained by an improvement in pelvis and hip motion, resulting in an increase in COM vertical displacement. Despite the normalization of mechanical work and muscular efficiency after surgery in patients with scoliosis, the energy cost remained exces- 
sive partly because of the absence of reduction in excessive time activity of the lumbo-pelvic muscles and possibly also due to morphological changes, such as residual growth of the lower limbs [23].

On the other hand, energy expenditure and endurance have an indirect correlation [38]. The results for the endurance test show that this parameter is significantly improved following revision surgery in revision group, but remains less than that in the primary and able-bodied groups. It would appear that the spinal deformity of the revision group had a considerable influence on walking endurance. However, it should be noted that diminished gait endurance is also related to cardiovascular and general fitness factors $[6,19]$. As a result, the corrective surgery permitted them to gain a substantial improvement in gait endurance following surgery. In addition, one study reported that the preoperative physical rehabilitation protocol would reveal a significant drop in RR and HR associated with an increase in the distance walked on the $6 \mathrm{MWT}$, which persists after corrective surgery. Inversely, patients with IS who do not undergo physical rehabilitation showed an increase in RR and HR [31]. Thus, these results suggest that patients trained with endurance exercises can show good performance even after surgery. However, further studies are needed to confirm this hypothesis.

Future studies should therefore include the following. (1) Investigation of the kinetic and EMG parameters should be performed in patients with IS following spinal surgery. (2) An investigation into the effect of surgery on the gait of patients with IS over a longer period will be beneficial. (3) Further clinical studies for specific overall body reconditioning and a training regimen should be conducted to evaluate whether excessive energy expenditure in walking is due to poor physical condition or muscular disease. (4) Evaluation of the cause of excessive energy expenditure in patients with IS following surgical intervention. (5) Further studies are needed, such as comparative studies including normal controls as well as high-quality RCTs, which is the best design to control for potential bias, and as a result, suggest the strongest evidence of cause-effect inferences between surgical intervention and outcomes in patients with IS.

\section{Conclusions}

Following revision surgery, ankle and foot kinematics do not change although pelvic and hip frontal motion increases and pelvic rotation decreases. It appears that rehabilitation strategies for improving gait velocity and endurance could be implemented to further improve the gait of these individuals. Surgery is, therefore, not disruptive for habitual functional activities, such as walking. However, postoperatively, patients with IS continued to show excessive energy expenditure in the absence of a physical rehabilitation protocol. There were inadequate data regarding the effect of corrective surgery on kinetics and EMG parameters.

\section{Conflict of Interest}

No potential conflict of interest relevant to this article was reported.

\section{References}

1. Weinstein SL, Dolan LA, Cheng JC, Danielsson A, Morcuende JA. Adolescent idiopathic scoliosis. Lancet 2008;371:1527-37.

2. Little DG, Song KM, Katz D, Herring JA. Relationship of peak height velocity to other maturity indicators in idiopathic scoliosis in girls. J Bone Joint Surg Am 2000;82:685-93.

3. Negrini S, Minozzi S, Bettany-Saltikov J, et al. Braces for idiopathic scoliosis in adolescents. Cochrane Database Syst Rev 2010;(1):CD006850.

4. Wang T, Xu JG, Zeng BF. Selective fusion in adolescent idiopathic scoliosis. Chin Med J (Engl) 2008;121:1456-61.

5. Wang T, Zeng B, Xu J, et al. Radiographic evaluation of selective anterior thoracolumbar or lumbar fusion for adolescent idiopathic scoliosis. Eur Spine J 2008;17:1012-8.

6. Engsberg JR, Bridwell KH, Reitenbach AK, et al. Preoperative gait comparisons between adults undergoing long spinal deformity fusion surgery (thoracic to L4, L5, or sacrum) and controls. Spine (Phila Pa 1976) 2001;26:2020-8.

7. Lenke LG, Engsberg JR, Ross SA, Reitenbach A, Blanke K, Bridwell KH. Prospective dynamic functional evaluation of gait and spinal balance following spinal fusion in adolescent idiopathic scoliosis. Spine (Phila Pa 1976) 2001;26:E330-7.

8. MacKinnon CD, Winter DA. Control of whole body 
balance in the frontal plane during human walking. J Biomech 1993;26:633-44.

9. Winter DA. Biomechanics and motor control of human gait: normal, elderly and pathological. 2nd ed. Waterloo (Ont): Waterloo Biomechanics; 1991.

10. Thorstensson A, Nilsson J, Carlson H, Zomlefer MR. Trunk movements in human locomotion. Acta Physiol Scand 1984;121:9-22.

11. Mahaudens P, Banse X, Mousny M, Detrembleur C. Gait in adolescent idiopathic scoliosis: kinematics and electromyographic analysis. Eur Spine J 2009;18:512-21.

12. Mahaudens P, Thonnard JL, Detrembleur C. Influence of structural pelvic disorders during standing and walking in adolescents with idiopathic scoliosis. Spine J 2005;5:427-33.

13. Mallau S, Bollini G, Jouve JL, Assaiante C. Locomotor skills and balance strategies in adolescents idiopathic scoliosis. Spine (Phila Pa 1976) 2007;32:E1422.

14. Giakas G, Baltzopoulos V, Dangerfield PH, Dorgan JC, Dalmira S. Comparison of gait patterns between healthy and scoliotic patients using time and frequency domain analysis of ground reaction forces. Spine (Phila Pa 1976) 1996;21:2235-42.

15. Mahaudens P, Detrembleur C, Mousny M, Banse X. Gait in adolescent idiopathic scoliosis: energy cost analysis. Eur Spine J 2009;18:1160-8.

16. Shiomi A. Idiopathic scoliosis by spinal fusion and brace treatment: evaluation by gait analysis. Nihon Seikeigeka Gakkai Zasshi 1995;69:665-74.

17. Sales de Gauzy J, Glorieux V, Dupui P, Montoya R, Cahuzac JP. Prospective gait analysis after surgery for idiopathic scoliosis. Orthop Proc 2008;90-B(Suppl 2):260.

18. Engsberg JR, Lenke LG, Uhrich ML, Ross SA, Bridwell KH. Prospective comparison of gait and trunk range of motion in adolescents with idiopathic thoracic scoliosis undergoing anterior or posterior spinal fusion. Spine (Phila Pa 1976) 2003;28:19932000.

19. Engsberg JR, Bridwell KH, Wagner JM, Uhrich ML, Blanke K, Lenke LG. Gait changes as the result of deformity reconstruction surgery in a group of adults with lumbar scoliosis. Spine (Phila Pa 1976) 2003;28:1836-43.

20. Wasylenko M, Skinner SR, Perry J, Antonelli DJ. An analysis of posture and gait following spinal fusion with Harrington instrumentation. Spine (Phila Pa 1976) 1983;8:840-5.

21. Paul JC, Patel A, Bianco K, et al. Gait stability improvement after fusion surgery for adolescent idiopathic scoliosis is influenced by corrective measures in coronal and sagittal planes. Gait Posture 2014;40:510-5.

22. Yagi $\mathrm{M}$, Ohne $\mathrm{H}$, Konomi $\mathrm{T}$, et al. Walking balance and compensatory gait mechanisms in surgically treated patients with adult spinal deformity. Spine J 2017;17:409-17.

23. Mahaudens P, Detrembleur C, Mousny M, Banse X. Gait in thoracolumbar/lumbar adolescent idiopathic scoliosis: effect of surgery on gait mechanisms. Eur Spine J 2010;19:1179-88.

24. Holewijn RM, Kingma I, de Kleuver M, Schimmel JJP, Keijsers NLW. Spinal fusion limits upper body range of motion during gait without inducing compensatory mechanisms in adolescent idiopathic scoliosis patients. Gait Posture 2017;57:1-6.

25. Holewijn R, Kingma I, de Kleuver M, Schimmel J, Keijsers N. Near preoperative shoulder and trunk range of motion during gait after surgical correction of adolescent idiopathic scoliosis. Global Spine J 2016;6(1 suppl):s-0036.

26. Raison M, Ballaz L, Detrembleur C, et al. Lombosacral joint efforts during gait: comparison between healthy and scoliotic subjects. Stud Health Technol Inform 2012;176:113-6.

27. Hopf C, Scheidecker M, Steffan K, Bodem F, Eysel P. Gait analysis in idiopathic scoliosis before and after surgery: a comparison of the pre- and postoperative muscle activation pattern. Eur Spine J 1998;7:6-11.

28. Dos Santos Alves VL, Stirbulov R, Avanzi O. Impact of a physical rehabilitation program on the respiratory function of adolescents with idiopathic scoliosis. Chest 2006;130:500-5.

29. Wallace J, King J, White H, Augsburger S, Milbrandt T, Iwinski H. A cross-sectional study of chest kinematics and VO2 in children with adolescent idiopathic scoliosis during steady-state walking. Spine (Phila Pa 1976) 2016;41:778-84.

30. Sperandio EF, Alexandre AS, Yi LC, et al. Functional aerobic exercise capacity limitation in adolescent idiopathic scoliosis. Spine J 2014;14:2366-72.

31. Dos Santos Alves VL, Stirbulov R, Avanzi O. Long- 
term impact of pre-operative physical rehabilitation protocol on the 6-min walk test of patients with adolescent idiopathic scoliosis: a randomized clinical trial. Rev Port Pneumol (2006) 2015;21:138-43.

32. Syczewska M, Graff K, Kalinowska M, Szczerbik E, Domaniecki J. Influence of the structural deformity of the spine on the gait pathology in scoliotic patients. Gait Posture 2012;35:209-13.

33. Yazdani S, Farahpour N. Spatiotemporal variables of gait in patients with adolescent idiopathic scoliosis and healthy individuals. J Sport Biomech 2014;1:3140.

34. Chen PQ, Wang JL, Tsuang YH, Liao TL, Huang PI, Hang YS. The postural stability control and gait pattern of idiopathic scoliosis adolescents. Clin Biomech (Bristol, Avon) 1998;13(1 Suppl 1):S52-8.
35. Della Croce U, Riley PO, Lelas JL, Kerrigan DC. A refined view of the determinants of gait. Gait Posture 2001;14:79-84.

36. Stoquart GG, Detrembleur C, Palumbo S, Deltombe T, Lejeune TM. Effect of botulinum toxin injection in the rectus femoris on stiff-knee gait in people with stroke: a prospective observational study. Arch Phys Med Rehabil 2008;89:56-61.

37. Inman VT, Ralston HJ, Todd F. Human walking. Baltimore (MD): Williams \& Wilkins; 1981.

38. Waters RL, Barnes G, Husserl T, Silver L, Liss R. Comparable energy expenditure after arthrodesis of the hip and ankle. J Bone Joint Surg Am 1988;70:1032-7. 